\title{
Chromosomal aberrations and prognosis in patients with concomitant chemoradiotherapy for resected head and neck cancer
}

\author{
SUNG-WON PARK ${ }^{1,2^{*}}$, YOUNG-HOON JOO ${ }^{3 *}$, SEUNG-HYUN JUNG ${ }^{1,2}$, SEON-HEE YIM ${ }^{1}$, \\ IN-CHUL NAM ${ }^{3}$, KWANG-JAE CHO ${ }^{3}$, MIN-SIK KIM ${ }^{3}$ and YEUN-JUN CHUNG ${ }^{1,2}$ \\ ${ }^{1}$ Integrated Research Center for Genome Polymorphism College of Medicine, The Catholic University of Korea, \\ Seocho-gu, Seoul 137-701; Departments of ${ }^{2}$ Microbiology and ${ }^{3}$ Otolaryngology-Head and Neck Surgery, \\ College of Medicine, The Catholic University of Korea, Seocho-gu, Seoul 137-040, Republic of Korea
}

Received August 10, 2015; Accepted October 6, 2015

DOI: $10.3892 / o r .2016 .4595$

\begin{abstract}
Although concomitant chemoradiotherapy (CCRT) has recently become a mainstay of a primary treatment modality in advanced head and neck squamous cell carcinoma (HNSCC), some of the patients experience CCRT failure. If we can predict the CCRT outcomes, we can reduce unnecessary CCRT avoiding risk of CCRT-related complication. We aimed to identify genetic alteration markers related to treatment failure in HNSCC patients who underwent radical surgery and CCRT. Genome-wide copy number alterations (CNAs) were analyzed in 18 HNSCC patients with $(n=9)$ or without $(n=9)$ recurrence using oligoarray-comparative genomic hybridization and candidate CNAs were validated by quantitative RT-PCR. A total of 15 recurrently altered regions (RARs) were identified in the 18 HNSCC cases. Among them, two RARs were significantly associated with CCRT-failure: copy number gained RARs of $7 \mathrm{p} 11.2$ harboring EGFR $(\mathrm{P}=0.029)$ and $18 \mathrm{p} 11.32$ harboring TYMS gene $(\mathrm{P}=0.029)$. Three RARs (7p11.2, 9p21.3 and 18p11.32) were significantly associated with poor disease-specific survival in univariate analysis, and 7 p11.2 was consistently significant in the multivariate analysis (HR 40.68, P=0.003). In conclusion, we defined novel genomic
\end{abstract}

Correspondence to: Professor Yeun-Jun Chung, Department of Microbiology, The Catholic University of Korea, College of Medicine, 222 Banpo-daero, Seocho-gu, Seoul 137-040, Republic of Korea E-mail: yejun@catholic.ac.kr

Dr Min-Sik Kim, Department of Otolaryngology-Head and Neck Surgery, Seoul St. Mary's Hospital, The Catholic University of Korea, 505 Banpo-dong, Seocho-gu, Seoul 137-040, Republic of Korea

E-mail: entkms@catholic.ac.kr

"Contributed equally

Key words: head and neck squamous cell carcinoma, concomitant chemoradiotherapy, treatment failure, array-CGH, copy number alteration alterations associated with CCRT-failure: 7p11.2 (EGFR) and 18p11.32 (TYMS). Our results provide useful clues for the elucidation of the molecular pathogenesis of HNSCC and to predict CCRT-failure.

\section{Introduction}

Head and neck squamous cell carcinoma (HNSCC) is one of the aggressive malignancies and a majority of the HNSCC patients belong to advanced stage with less than $50 \%$ of long-term survival (1). Treatment of advanced-stage HNSCC has undergone substantial changes in recent years. Combined modality treatments including radical surgery and various regimens of concomitant chemoradiotherapy (CCRT) have been used to improve locoregional control and distant metastases of the cancer (1). However, even though CCRT has recently become a mainstay of a primary treatment modality in advanced HNSCC, some of the patients may experience cancer recurrence and CCRT protocols can lead to some serious complications such as swallowing dysfunction resulting in permanent gastrostomy tube dependence (2). Therefore, if we can distinguish the patients who would benefit from CCRT after the surgery, from those that will not, before applying it, we would reduce unnecessary CCRT thus avoiding risk of CCRT-related complication.

Several molecular-genetic factors have been suggested to be potentially contributing to the resistance to CCRT. For example, CCRT-resistant HNSCCs showed a high rate of p53 aberrations and increased expression of MDM2, which confers tumor cell survival after CCRT-induced cytotoxic stress $(3,4)$. Expression levels of hMLH1 and RKIP were also reported to be predictive of responsiveness to CCRT in head and neck cancers $(5,6)$. Clinical factors such as the serum LDH and hemoglobin levels have been suggested as prognostic factors after CCRT $(7,8)$.

Chromosomal copy number alteration (CNA), both amplification and deletion, is one of the hallmarks of cancer including HNSCC. Amplifications of the genes involved in detoxification of chemotherapeutic agents or deletions of the genes involved in DNA repair have been reported to 
be associated with CCRT-resistance (9). More specifically, gains of 3q11-q13, 3q21-q26.1 and 6q22-q27, and losses of 3 p11-pter and 4p11-pter were reported to be significantly associated with CCRT resistance in HNSCC by comparative genomic hybridization (CGH) analysis (10). However, due to its limited resolution, the information from $\mathrm{CGH}$ analyses has not been detailed enough to identify the causative genes. Two genome-wide analyses, microarray based CGH (array-CGH) and next generation sequencing (NGS), are currently the best tools with much improved resolution for detecting CNAs precisely. In terms of detecting CNAs, it is widely accepted that array-CGH is relatively more matured and cost-effective technology than NGS (11). However, there has been no report exploring CNA profiles in HNSCCs with CCRT and their prognostic implications through high resolution array-CGH.

To explore genetic alterations predicting the responsiveness to radical surgery plus postoperative CCRT in HNSCC patients, we analyzed the CNAs across the whole genome using oligoarray-CGH for the HNSCC patients who had been administered postoperative CCRT.

\section{Materials and methods}

Patients and tumor samples. We retrospectively selected 18 patients who were diagnosed with HNSCC and underwent primary tumor resection and neck dissection followed by CCRT at the Department of Otolaryngology-Head and Neck Surgery from April 2008 to June 2012. Tumor stage was determined according to the standard tumor-node-metastasis classification of AJCC guidelines (7th edition). Frozen tissues (tumor and adjacent normal tissue pairs) were obtained from the patients with previously untreated HNSCC. Indications for adjuvant CCRT are as follows: positive or close margins found on the resection, advanced $\mathrm{T}$ stage, lymphovascular invasion, perineural invasion, multiple nodal metastasis, or extracapsular spread. All $18 \mathrm{HNSCC}$ patients received postoperative radiotherapy (RT) that consisted of conventionally fractionated doses of 1.8-2 Gy each in five weekly sessions. A volume including the primary tumor site and all the draining lymph nodes at risk received a dose of 50-55 Gy. Regions that had an inadequate resection margin, extracapsular nodal spread or the initially involved lymph node area were boosted up to $65 \mathrm{~Gy}$. The patients received chemotherapy that consisted of $100 \mathrm{mg}$ cisplatin $/ \mathrm{m}^{2}$ of body-surface area on days 1,22 and 43 during RT or weekly $30 \mathrm{mg}$ cisplatin $/ \mathrm{m}^{2}$ of body surface area. The Institutional Review Board of Seoul St. Mary's Hospital approved the retrospective review of the medical records and the use of archived tumor specimens.

Array comparative genomic hybridization. For array-CGH analysis, $10-\mu \mathrm{m}$-thick frozen sections of tumor cell-rich areas (>60\%) were microdissected. Genomic DNA was extracted from these sections using a DNeasy Blood and Tissue kit (Qiagen, Hilden, Germany). The Agilent SurePrint G3 Human CGH Microarray kit, 4x180K (Agilent technologies, Santa Clara, CA, USA) was used for the array-CGH analysis. This array contains 180,880 probes with a median probe spacing of $13 \mathrm{~kb}$. The array hybridization procedure was performed as described elsewhere (12). In brief, $1 \mu \mathrm{g}$ of genomic DNA (gDNA) from tumor tissue was labeled with Cy5-dCTP and $1 \mu \mathrm{g}$ of gDNA from paired normal tissue with Cy3-dCTP. After purifying the labeled product by using Amicon Ultra-0.5 centrifugal Filter Device (Millipore, Billerica, MA, USA), the labeled DNA was applied on the Agilent 4x180K array with the blocking agent (Agilent technologies, Santa Clara, CA, USA) containing $50 \mu \mathrm{g}$ of human Cot-1 DNA (HybMasker, ConnectaGen, Korea). Array slides were incubated for $24 \mathrm{~h}$ at $65^{\circ} \mathrm{C}$ in the hybridization oven. After the hybridization, the arrays were washed with wash buffer 1 and 2 according to the manufacturer's protocol and scanned using High-Resolution Microarray Scanner (Agilent technologies).

Determination of CNAs and recurrently altered regions. Microarray hybridization images were analyzed with Feature Extraction Software version 10.7.3.1 using CGH-107-Sep09 protocol for normalization as described previously (12). Data quality was assessed using CGH_QCTM_Sep09 QC metric set. Only the data that passed the quality check (in good or excellent range) was used for CNA calling. CNAs of samples were defined using the ADM-2 algorithm in Agilent Genomic Workbench Lite Edition 6.0 software (Agilent technologies). CNAs were defined using the following criteria; a minimum number of probes in region $=3$, a maximum number of probes in aberrant region $=100,000$, a signal intensity ratio of $>0.3$ in $\log _{2}$ scale for gains, and of $<-0.3$ in $\log _{2}$ scale for losses. High-level amplification was defined as a probe signal intensity ratio of 1 or higher in $\log _{2}$ scale. Likewise, a homozygous deletion (HD) was defined as a ratio of -1 or lower in $\log _{2}$ scale. After defining CNAs, recurrently altered regions (RAR) were defined using the CNVRuler program as chromosomal segments consisting of multiple overlapping CNAs found in $\geq 30 \%$ of the samples (13).

Real-time quantitative PCR ( $P$ PCR) analysis. We performed qPCR validation of candidate RARs using the gDNA extracted from 18 pairs of head and neck cancer/normal tissues. As a diploid internal control, we used a genomic region on chromosome 13 (13q32.1) which was observed to have no genomic alteration in the array-CGH data. Sequence and location of primers used for the genomic qPCR experiment are as follows. RAR-G2 (7p12.1-p11.2 containing EGFR) forward, TTTGCC AAGGCACGAGTAA and reverse, CAAGGACCACCTCAC AGTTATT; RAR-G8 (18p11.32 containing TYMS) forward, ATGTGGTGAACAGTGAGCTGTCCT and reverse, AATCA TGTACGTGAGCAGGGCGTA; RAR-L4 (9p21.3 containing $C D K N 2 A / B)$ forward, TTTGGAGGACAAGCTGGTGCA ATG and reverse, TCCCAGATGAGGACAATGAGGCAA; Diploid control (16p12.2), forward, GACCTACCCACTTTCA ACTCTG and reverse, CTCTCCAAGCGCAAGGTATT. Genomic qPCR were performed using ViiA 7 Real-Time PCR System and ViiA 7 RUO software (Applied Biosystems, Foster City, CA, USA) as described previously (14). In brief, qPCR mixture of $10 \mu \mathrm{l}$ contains $10 \mathrm{ng}$ of gDNA, Maxima ${ }^{\circledR}$ SYBR-Green qPCR Master Mix (2X), ROX solution provided (Fermentas, Foster, CA, USA), and 5 pmol primers. Thermal cycling conditions consist of one cycle of $10 \mathrm{~min}$ at $95^{\circ} \mathrm{C}$, followed by 40 cycles of $5 \mathrm{sec}$ at $95^{\circ} \mathrm{C}, 10 \mathrm{sec}$ at $61^{\circ} \mathrm{C}$ and $20 \mathrm{sec}$ at $72^{\circ} \mathrm{C}$. The dissociation value of the amplified PCR product was measured as the temperature inched up at a rate of $0.1^{\circ} \mathrm{C} / \mathrm{sec}$ from $55^{\circ} \mathrm{C}$ to $95^{\circ} \mathrm{C}$. All qPCR experiments were 
Table I. Clinicopathological characteristics of the 18 HNSCC patients.

\begin{tabular}{|c|c|}
\hline Parameter & No. of patients (\%) \\
\hline \multicolumn{2}{|l|}{ Age (years) } \\
\hline$\geq 60$ & $7(38.9)$ \\
\hline$<60$ & $11(61.1)$ \\
\hline \multicolumn{2}{|l|}{ Gender } \\
\hline Male & $18(100)$ \\
\hline Female & $0(0)$ \\
\hline \multicolumn{2}{|l|}{ Primary tumor } \\
\hline Oral cavity & $2(11.1)$ \\
\hline Oropharynx & $8(44.4)$ \\
\hline Larynx & $3(16.7)$ \\
\hline Hypopharynx & $5(27.8)$ \\
\hline \multicolumn{2}{|l|}{ T stage } \\
\hline $\mathrm{T} 1$ & $1(5.6)$ \\
\hline $\mathrm{T} 2$ & $11(61.1)$ \\
\hline $\mathrm{T} 3$ & $6(33.3)$ \\
\hline \multicolumn{2}{|l|}{ Cervical lymph node stage } \\
\hline No & $2(11.1)$ \\
\hline N1 & $4(22.2)$ \\
\hline $\mathrm{N} 2$ & $11(61.1)$ \\
\hline N3 & $1(5.6)$ \\
\hline \multicolumn{2}{|l|}{ Histologic grade } \\
\hline Well differentiated & $2(11.1)$ \\
\hline Moderate differentiated & $15(83.3)$ \\
\hline Poorly differentiated & $1(5.6)$ \\
\hline \multicolumn{2}{|l|}{ Lymphovascular invasion } \\
\hline Yes & $13(72.2)$ \\
\hline No & $5(27.8)$ \\
\hline \multicolumn{2}{|l|}{ Extracapsular spread } \\
\hline Yes & $9(50.0)$ \\
\hline No & $9(50.0)$ \\
\hline \multicolumn{2}{|l|}{ Margin involvement } \\
\hline Yes & $3(16.7)$ \\
\hline No & $15(83.3)$ \\
\hline \multicolumn{2}{|l|}{ Recurrence } \\
\hline Yes & $9(50.0)$ \\
\hline No & $9(50.0)$ \\
\hline
\end{tabular}

triplicated and relative quantification was performed by the $2^{-\Delta \Delta C T}(15)$. Relative ratios were used to determine whether the status of a CNA is copy number gain $(>2)$ or loss $(<0.5)$.

Statistical analysis. To explore associations of chromosomal copy number changes and clinicopathologic phenotypes, phenotype variables were treated as categorical variables, that is, age, gender, primary site, histologic grade, T stage, cervical lymph node metastasis, presence or absence of lymphovascular invasion, extracapsular spread and recurrence. Differences in the distributions of RARs for each categorical variable were tested using two-sided Fisher's exact test. Disease-specific survival was determined using the Kaplan-Meier method. The Cox proportional hazards model with likelihood ratio statistics was used to identify variables significantly and independently related to survival. $\mathrm{P}<0.05$ was considered to indicate a statistically significant difference, and all calculations were performed using SPSS version 16.0 (SPSS, Chicago, IL, USA).

\section{Results}

Clinicopathological characteristics. The median age was 59.2 years (range, 49-77 years) and all patients were males. The sites of the original primary tumor were oral cavity $(11.1 \%)$, oropharynx $(44.4 \%)$, larynx $(16.7 \%)$ and hypopharynx (27.8\%). A majority of them $(17 / 18,94.4 \%)$ were stage $\mathrm{T} 2$ and $\mathrm{T} 3$, and sixteen patients $(89.0 \%)$ were cervical lymph node positives. Thirteen patients (72.2\%) were lymphovascular invasion positives and nine (50.0\%) had extracapsular spread of a metastatic neck node. A total of nine patients (50.0\%) had tumor recurrence after CCRT. There was no clinicopathological factors significantly associated with the treatment failure defined as tumor recurrence. Details of clinicopathological characteristics of the study subjects are summarized in Table I.

Copy number alteration profiles in HNSCC. The overall frequency plot of the CNAs detected in the 18 HNSCCs (9 with and 9 without recurrence) showed that CNAs were not randomly distributed but rather clustered in certain chromosomal segments across the genome (Fig. 1A). The mean number of CNAs per each HNSCC genome was 41.3 \pm 18.6 . Patients with recurrence had more CNAs than those without recurrence (mean frequency $57.3 \pm 24.9$ vs. $25.3 \pm 7.2$ ), but the difference did not reach statistical significance. As a whole, 8 chromosomal arms were recurrently (appeared in $\geq 2$ cases) gained on: $2 p(11 \%), 3 q$ (28\%), 5p (11\%), 7p (11\%), 8q (11\%), $12 \mathrm{p}(11 \%), 18 \mathrm{p}(11 \%)$ and 22q (11\%). Also, 8 chromosomal arms were recurrently deleted: $1 \mathrm{p}(11 \%), 3 \mathrm{p}(22 \%), 4 \mathrm{p}(11 \%)$, $5 q(22 \%), 10 p(17 \%), 13 q(17 \%), 14 q(11 \%)$ and 21q (17\%). Of the recurrent chromosomal arm changes, copy number gains on $7 p, 8 q$ and $18 p$ were observed only in the treatment failure group but these differences were not statistically significant.

Recurrently altered chromosomal regions. In addition to the entire chromosomal arm changes, many of the CNAs were regional changes. Some of them were observed recurrently, suggesting their potential contribution to tumorigenesis and progression. To identify the commonly implicated CNAs, we defined RARs. In the present study, chromosomal segments as the union of overlapping CNAs in $\geq 20 \%$ of the 18 HNSCCs were defined as RARs (RAR-G for gains and RAR-L for losses, respectively). A total of 15 RARs (8 RAR-Gs and 7 RAR-Ls) were identified in the 18 HNSCC patients (Table II). Fig. 2 illustrates the RAR-G on 7p11.2 and RAR-L on 9p21.3 as examples. Of the 15 RARs, a RAR-G on 7 p11.2 (RAR-G2: $4.22 \mathrm{Mb}$-sized, 55.6\% in recurrence group vs. 0\% in non-recurrence group, $\mathrm{P}=0.029$ ), and a RAR-G on $18 \mathrm{p} 11.32$ (RAR-G8: $4.35 \mathrm{Mb}$-sized, $44.4 \%$ in recurrence group vs. $0 \%$ in non-recurrence group, $\mathrm{P}=0.029$ ) were significantly more common in the HNSCC with recurrence group than in the HNSCC without recurrence group. The well-known 
A

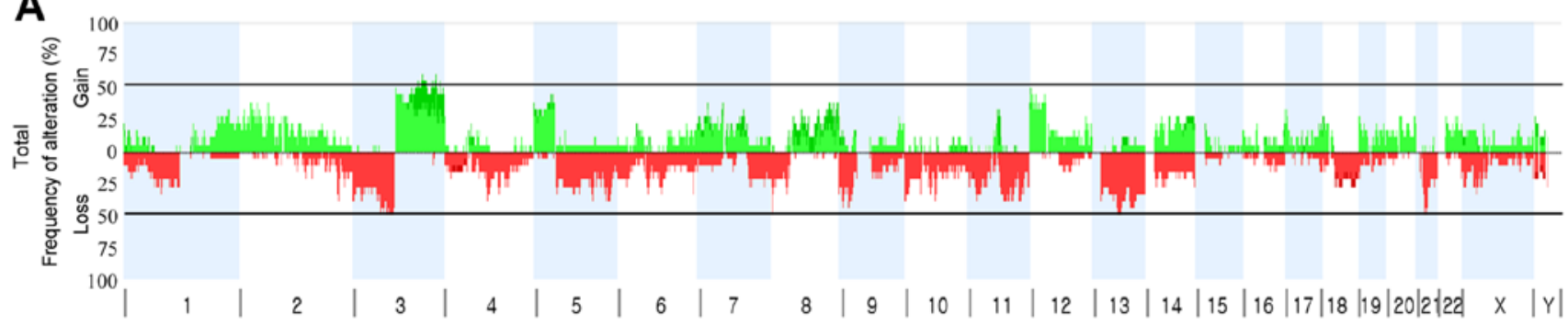

B
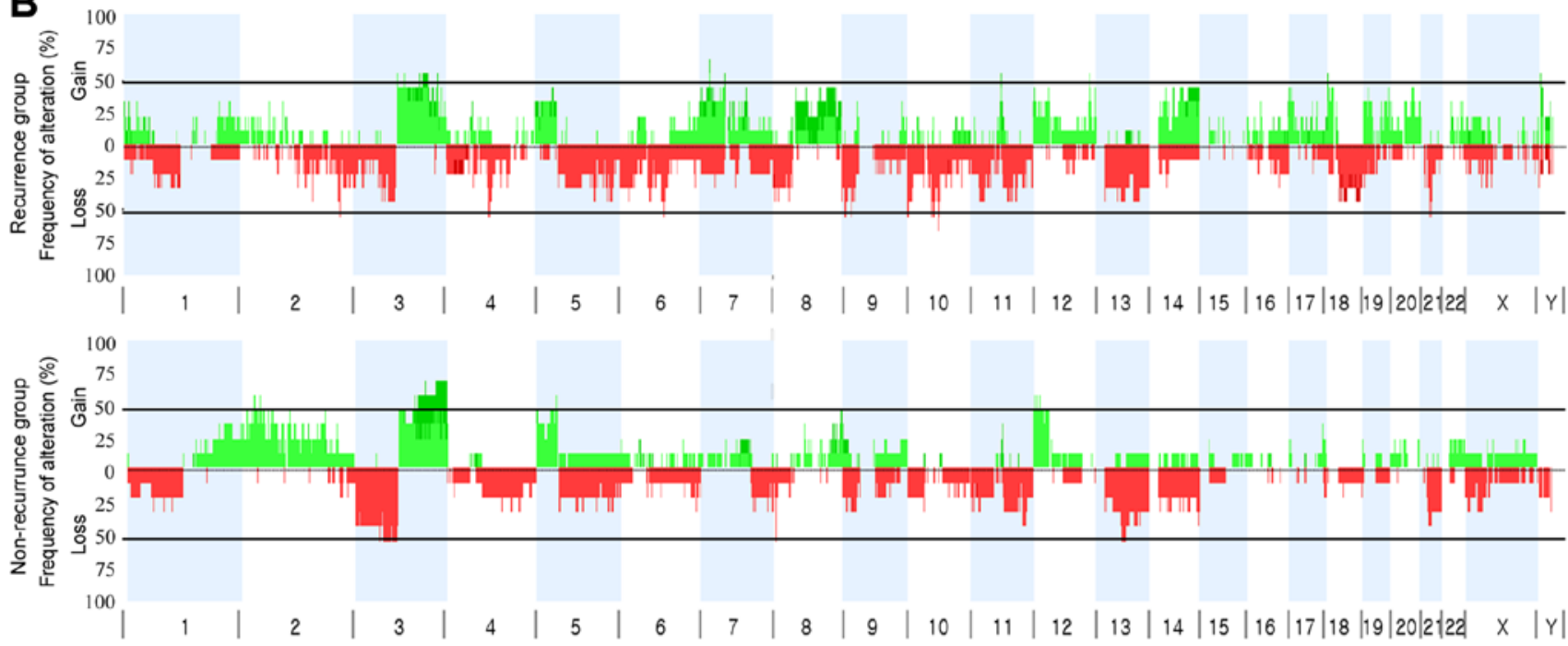

Figure 1. Genome-wide profiles and frequency plot of chromosomal alterations and number of CNAs in 18 HNSCC. Total frequencies of copy number gains and losses in 18 HNSCCs (A) and frequency of alteration in recurrence (upper plot) or non-recurrence (lower plot) after CCRT (B). The green bars denote the copy number gains and the red bars denote the copy number losses. Boundaries of individual chromosomes are indicated by vertical bars.
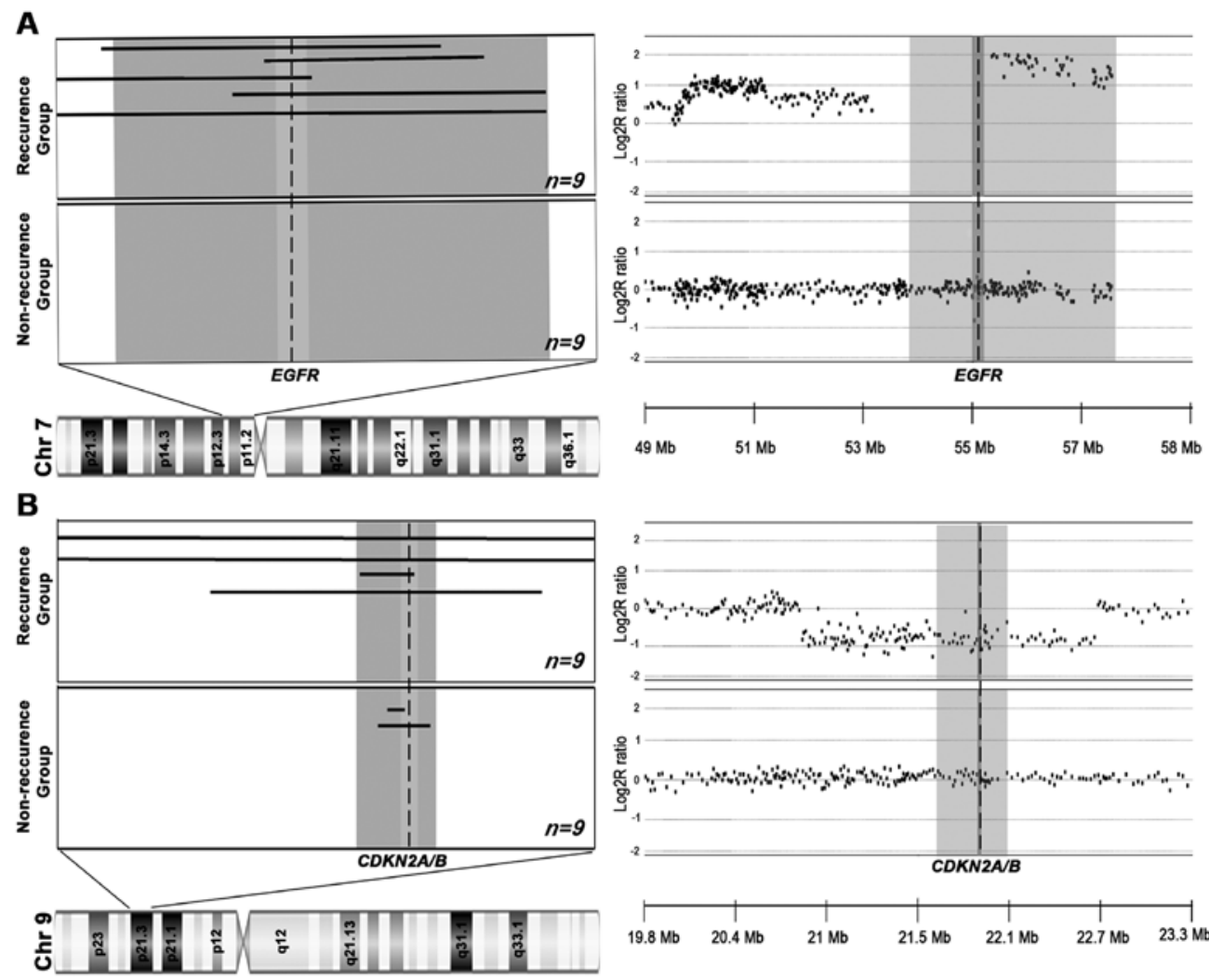

Figure 2. Examples of recurrently altered regions. RAR-G on 7p11.2 (A) and RAR-L on 9p21.3 (B). Upper plot illustrates the recurrence group and lower plot illustrates non-recurrence group after CCRT. Black bars, CNAs of each HNSCC. Gray areas, RARs on 7p11.2 and 9p21.3. Yellow areas, EGFR and $C D K N 2 A / B$ gene loci in those RARs. 


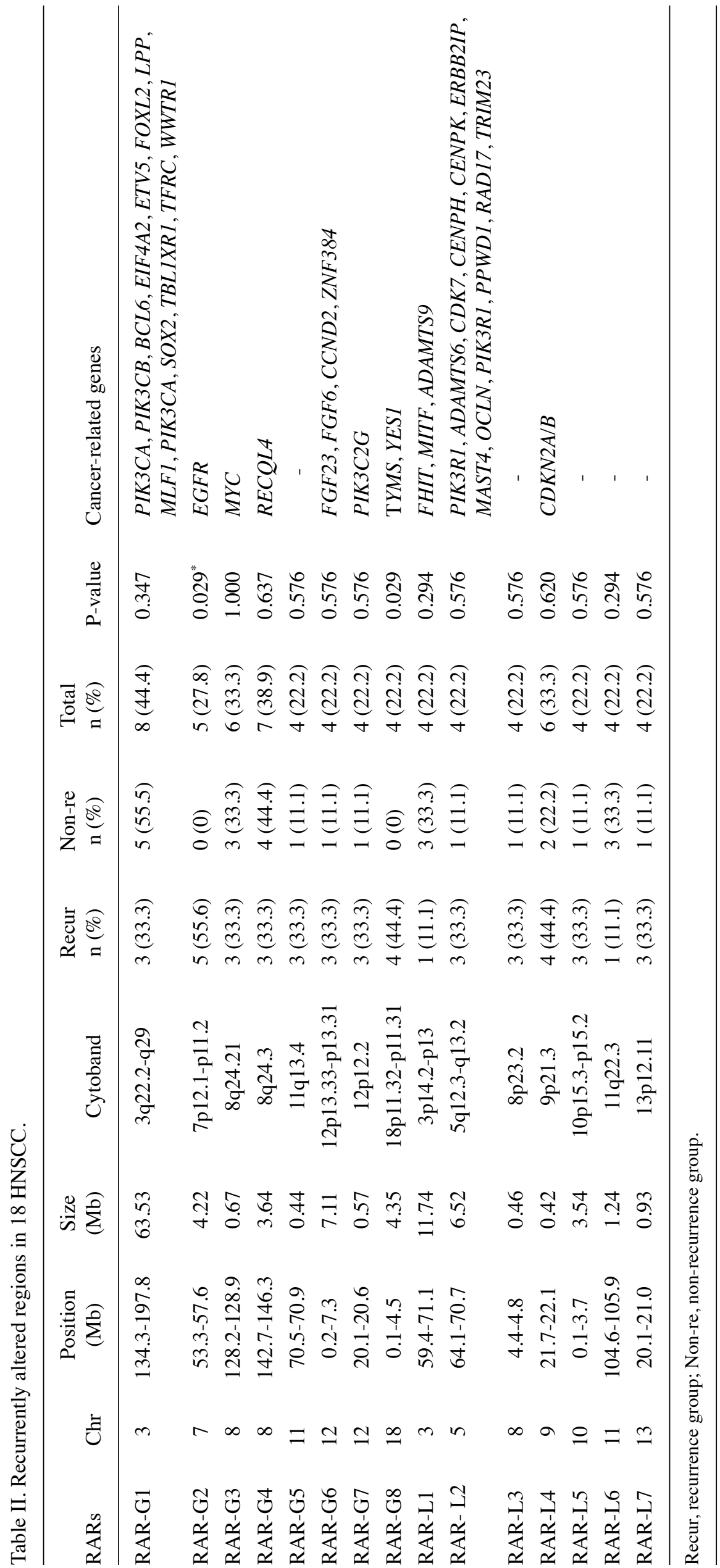


Table III. High-level copy number changes in HNSCC.

\begin{tabular}{|c|c|c|c|c|c|c|c|c|}
\hline CNAs & $\mathrm{Chr}$ & $\begin{array}{l}\text { Position } \\
\text { (MP) }\end{array}$ & $\begin{array}{l}\text { Size } \\
(\mathrm{Mb})\end{array}$ & Cytoband & $\begin{array}{l}\text { Freq \% } \\
\text { (Recur) }\end{array}$ & $\begin{array}{l}\text { Freq \% } \\
\text { (Non-re) }\end{array}$ & $\begin{array}{l}\text { Freq \% } \\
\text { (Total) }\end{array}$ & $\begin{array}{c}\text { Cancer-related } \\
\text { genes }\end{array}$ \\
\hline Amp1 & 1 & $59.96-61.93$ & 1.97 & p32.1-p31.3 & 11.1 & 0.0 & 11.1 & - \\
\hline Amp2 & 3 & $181.62-183.18$ & 1.56 & q26.33-q27.1 & 11.1 & 0.0 & 11.1 & - \\
\hline Amp3 & 7 & 54.87-56.57 & 1.70 & p11.2 & 22.2 & 0.0 & 22.2 & EGFR \\
\hline Amp4 & 7 & $64.10-66.63$ & 2.53 & $\mathrm{q} 11.21$ & 11.1 & 0.0 & 11.1 & $S B D S$ \\
\hline Amp5 & 10 & $35.15-36.00$ & 0.85 & $\mathrm{p} 11.21$ & 0.0 & 11.1 & 11.1 & - \\
\hline Amp6 & 10 & $55.04-55.13$ & 0.09 & $\mathrm{q} 21.1$ & 11.1 & 0.0 & 11.1 & - \\
\hline Amp7 & 11 & $69.48-69.57$ & 0.09 & q13.3 & 22.2 & 11.1 & 33.3 & $C C N D 1$ \\
\hline Amp8 & 11 & $84.35-84.55$ & 0.20 & $\mathrm{q} 14.1$ & 0.0 & 11.1 & 11.1 & - \\
\hline Amp9 & 11 & $100.89-101.44$ & 0.55 & $\mathrm{q} 22.1$ & 0.0 & 11.1 & 11.1 & - \\
\hline Amp10 & 13 & $64.76-67.46$ & 2.70 & $\mathrm{q} 21.31-\mathrm{q} 21.32$ & 11.1 & 0.0 & 11.1 & - \\
\hline Amp11 & 13 & $73.64-74.37$ & 0.73 & $\mathrm{q} 22.1$ & 11.1 & 0.0 & 11.1 & - \\
\hline Amp12 & 13 & $77.48-78.10$ & 0.62 & $\mathrm{q} 22.3$ & 11.1 & 0.0 & 11.1 & - \\
\hline Amp13 & 16 & $51.03-53.37$ & 2.34 & $\mathrm{q} 12.1-\mathrm{q} 12.2$ & 0.0 & 11.1 & 11.1 & - \\
\hline Amp14 & 17 & $46.30-46.99$ & 0.69 & $\mathrm{q} 21.32$ & 11.1 & 0.0 & 11.1 & - \\
\hline Amp15 & 18 & $0.12-4.47$ & 4.35 & p11.32 & 11.1 & 0.0 & 11.1 & - \\
\hline HD1 & 1 & 72.77-72.80 & 0.03 & p31.1 & 11.1 & 11.1 & 22.2 & - \\
\hline HD2 & 2 & $141.17-141.27$ & 0.10 & $\mathrm{q} 22.1$ & 11.1 & 0.0 & 11.1 & - \\
\hline HD3 & 3 & $4.13-4.49$ & 0.36 & p26.1 & 11.1 & 0.0 & 11.1 & - \\
\hline HD4 & 3 & $162.51-162.62$ & 0.10 & $\mathrm{q} 26.1$ & 11.1 & 0.0 & 11.1 & - \\
\hline HD5 & 4 & 69.39-69.46 & 0.07 & q13.2 & $11 . .1$ & 11.1 & 22.2 & - \\
\hline HD6 & 5 & $81.35-81.52$ & 0.17 & $\mathrm{q} 14.1-\mathrm{q} 14.2$ & 0.0 & 11.1 & 11.1 & - \\
\hline HD7 & 8 & $2.76-3.18$ & 0.42 & $\mathrm{p} 23.2$ & 11.1 & 0.0 & 11.1 & - \\
\hline HD8 & 9 & 21.96-22.03 & 0.07 & p21.3 & 22.2 & 11.1 & 33.3 & $C D K N 2 A$ \\
\hline HD9 & 10 & $88.47-90.75$ & 2.28 & $\mathrm{q} 23.2-\mathrm{q} 23.31$ & 11.1 & 0.0 & 11.1 & BMPRIA, PTEN \\
\hline HD10 & 11 & $12.32-12.94$ & 0.62 & $\mathrm{p} 15.3-\mathrm{p} 15.2$ & 0.0 & 11.1 & 11.1 & - \\
\hline
\end{tabular}

Amp, amplification; HD, homozygous deletion; Recur, recurrence group; Non-re, non-recurrence group. Bold, total frequency $>20 \%$.

oncogene EGFR (7p11.2) and the potential oncogene $T Y M S$ (18p11.32) are located in the recurrence-group dominant RAR-Gs. A RAR-L on 9p21.3 (0.42 Mb-sized RAR-L4), where CDKN2A/B are located, more commonly occurred in the HNSCC with recurrence group (44.4\%) than in non-recurrence group $(22.2 \%)$ but it was not statistically significant. To validate the three recurrence group dominant RARs identified by array-CGH, we performed genomic qPCR validations for those loci (7p11.2, 9p21.3 and 18p11.32). All the CNAs identified by array-CGH were consistently detected by the qPCR and as a whole $93.9 \%$ (46/49) of the qPCR results were consistent with the copy number status by array-CGH (data not shown).

High-level CNAs in HNSCC. Of the CNAs identified in the 18 HNSCCs, 25 loci were found to be high-level CNAs (defined as $\geq 1$ for amplification or $\leq-1$ for HD on the $\log _{2}$ scale), with 15 amplifications and 10 HDs (Table III). Five high-level CNAs were detected in $>20 \%$ of the samples; amplifications on $7 \mathrm{p} 11.2$ and 11q13.3, and HDs on 1p31.1, 4q13.2 and 9p21.3, respectively (Table III). Well-known oncogenes (EGFR and
$C C N D 1)$ and tumor suppressor gene $(C D K N 2 A / B)$ are located in the recurrent $(>20 \%)$ high-level CNA regions.

RARs associated with the prognosis in HNSCC. Univariate survival analysis was performed to assess the implications of the RARs on patient survival. Univariate analysis was also performed to identify clinicopathologic features (age, gender, primary site, histologic grade, $\mathrm{T}$ stage, cervical lymph node metastasis, lymphovascular invasion, extracapsular spread and recurrence) for inclusion as covariates for Cox regression. Five-year disease-specific survival rates (DSSR) in our cohort were $66 \%$. Among the RARs and clinicopathologic factors, cervical lymph node metastasis $(\mathrm{P}=0.008)$, recurrence $(\mathrm{P}=0.003)$, RAR-G2 ( $<<0.001)$, RAR-G8 ( $<<0.001)$, and RAR-L4 ( $\mathrm{P}=0.037)$ were significantly associated with poor DSSR in the univariate analysis (Fig. 3). Multivariate analysis with the three significant RARs and two clinical variables identified on univariate analysis revealed that RAR-G2 [hazard ratio $(\mathrm{HR})=40.68$, 95\% confidence interval $(\mathrm{CI})$ : 3.56-464.69, $\mathrm{P}=0.003$ ] and cervical metastasis $(\mathrm{HR}=16.38,95 \% \mathrm{CI}: 1.20-223.11, \mathrm{P}=0.036)$ were significantly associated with poor DSSR (Table IV). 
A
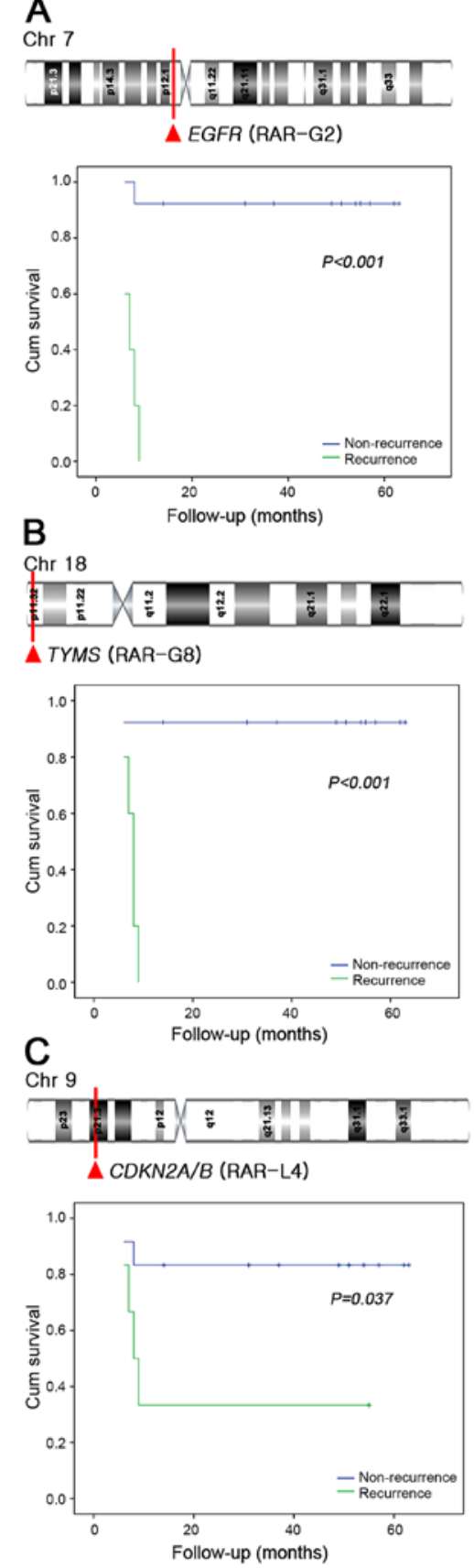

Figure 3. Kaplan-Meier curves by RAR status in the univariate analysis. RAR-G on 7p11.2 harboring EGFR gene (A), RAR-G on 18p11.32 harboring TYMS gene (B) and RAR-L on 9p21.3 harboring $C D K N 2 A / B(\mathrm{C})$.

Table IV. Results of multivariate analysis.

\begin{tabular}{lccrr}
\hline & & \multicolumn{2}{c}{$95 \% \mathrm{CI}$} & \\
\cline { 3 - 4 } Variables & HR & Lower & Upper & P-value \\
\hline Cervical metastasis & 16.38 & 1.20 & 223.11 & 0.036 \\
Recurrence & 0.940 & 0.00 & 17.30 & 0.332 \\
RAR-G02 & 40.68 & 3.56 & 464.69 & 0.003 \\
RAR-G08 & 2.16 & 0.16 & 22.46 & 0.142 \\
RAR-L04 & 1.21 & 0.00 & 7.76 & 0.217 \\
\hline
\end{tabular}

HR, hazard ratio; CI, confidence interval.

\section{Discussion}

Despite the various treatment modalities, the poor prognosis of HNSCC has not improved significantly over the last four decades (16). The lack of success in HNSCC treatment may be primarily due to lack of understanding on the etiology and heterogeneous nature of HNSCC. In the present study, we aimed to explore CNA profiles associated with the resistance after CCRT in HNSCCs. For this, we analyzed the CNAs using high-resolution array-CGH (180K oligoarray) and delineated RARs under the assumption that commonly altered chromosomal segments in CCRT-resistant subgroup may contain essential genes contributing to CCRT resistance. Through this approach, we identified the genetic markers predicting the responsiveness to CCRT following surgery in HNSCC patients.

The key alterations identified in the present study, such as amplifications of PIK3CA, EGFR and MYC and deletions of $C D K N 2 A / B$ and $F H I T$, were consistent with previous observations in HNSCC, suggesting the reliability of our study $(10,12,17,18)$. We also identified several novel RARs which have not been reported to be associated with HNSCC previously; RAR-Gs in 12p13 and 12p12 harboring FGF6, FGF23 and PIK3C2G genes and a RAR-L in $8 \mathrm{p} 23.2$ harboring CSMDI gene. To validate the array-CGH results, we performed genomic qPCR validation for the three interesting RARs (RAR-G2, RAR-G8 and RAR-L4) identified by array-CGH and all of the CNAs were consistently defined in the qPCR, suggesting the reliability of our array-CGH analysis and advantage of higher resolution array analysis.

Two RARs, RAR-G2 (7p11.2 encompassing EGFR) and RAR-G8 (18p11.32 encompassing TYMS), were significantly more common in the HNSCC patients with recurrence (CCRT non-responders) than in those without recurrence (CCRT-responders). In the observations of Gao et al, EGFR overexpression was associated with poor survival in HNSCC patients receiving CCRT (19). Szabó et al examined expression and genetic alteration of $E G F R$ gene by FISH with 71 HNSCC patients and observed that increased copy number and overexpression of the EGFR gene was associated with poor prognosis in HNSCC (20). However, neither group examined the CNAs involving the responsiveness to CCRT in terms of recurrence. To our knowledge, this is the first report which explored the CNA profiles across the whole genome in HNSCC using high-resolution array-CGH and demonstrated that the copy number gain of $17 \mathrm{p} 11.2$ containing EGFR was significantly associated with the CCRT failure. When we assessed the prognostic implications of RARs, the RAR-G2 containing $E G F R$ was the only significantly associated with poor DSSR in multivariate analysis. Previously, increased copy number of $E G F R$ gene was also strongly associated with poorer survival, both overall and recurrence-free, in HNSCC (21).

In addition to the EGFR, the copy number gain of TYMS (RAR-G8), which codes thymidylate synthase, was also found to be significantly more common in the CCRT non-responders. TYMS catalyzes the conversion of dUMP to dTMP, which is essential for DNA biosynthesis (22). Consistent with our finding, overexpression of TYMS, caused by gene amplification, has been reported to be associated with poor prognosis and resistance to chemotherapeutic agents that target thymi- 
dylate biosynthesis such as 5-FU (23-25). In the study of Yasumatsu et al exploring the effect of TYMS knockdown in a HNSCC cell line (YCU-N), repressed expression of TYMS increased the cytotoxicity of 5-FU and inhibited cell proliferation (26). These data consistently suggest the implication of copy number gain of TYMS in acquiring resistance to 5-FU, which may contribute to the resistance to CCRT.

In this study, a copy number loss of $0.46 \mathrm{Mb}$-sized locus in 8p23.2, where CSMD1 gene is located, were recurrently observed in the HNSCC patients. The deletion of 8p23 locus has been frequently reported in HNSCCs and through the deletion mapping analysis, CSMD1 gene in this locus has been suggested as a tumor suppressor (27-29). The deletion of CSMDI has been also frequently reported in diverse solid tumors and the underexpression of $C S M D 1$ was reported to be associated with poor prognosis or higher tumor grade (30-32). Although copy number loss of CSMD1 gene did not show significant association with CCRT-resistance in our study, there was a tendency of non-responder dominance ( 3 in non-responders vs. 1 in responders). To our knowledge, this is the first report to identify the frequent copy number loss of CSMDI gene in HNSCC with CCRT resistance. Larger scale analysis will be required to verify this possibility.

In spite of the interesting findings, there were several limitations to the present study. First of all, due to the limited number of patients treated with primary surgery plus CCRT, some potential CCRT-associated CNAs could be missed. Second, the median follow-up period of this study was relatively short. Prospective, randomized studies using a larger sample will be required to assess the implications of the CNAs identified in the present study on clinical results following different treatment modalities.

In conclusion, we successfully defined novel recurrent genomic alteration regions and known copy number changes in 18 HNSCC with a well-designed high-resolution array CGH. Copy number gain of EGFR (RAR-G2) and TYMS (RAR-G8) were associated with CCRT failure and RAR-G2 was also found to be an independent factor of poor DSSR in HNSCC. Our results and the strategy of analysis will provide useful clues for further studies to elucidate the molecular pathogenesis of HNSCC and to develop biomarkers for designing personalized treatment by predicting the prognosis.

\section{Acknowledgements}

The present study was supported by the Cancer Evolution Research Center (grant no. 2012R1A5A2047939) and the Institute of Clinical Medicine Research of Bucheon St. Mary's Hospital Research Fund, 2015.

\section{References}

1. Pignon JP, Bourhis J, Domenge C and Designé L: Chemotherapy added to locoregional treatment for head and neck squamous-cell carcinoma: Three meta-analyses of updated individual data. MACH-NC Collaborative Group. Meta-Analysis of Chemotherapy on Head and Neck Cancer. Lancet 355: 949-955, 2000.

2. Smith RV, Kotz T, Beitler JJ and Wadler S: Long-term swallowing problems after organ preservation therapy with concomitant radiation therapy and intravenous hydroxyurea: Initial results. Arch Otolaryngol Head Neck Surg 126: 384-389, 2000.
3. Cinelli M, Magnelli L and Chiarugi V: Redundant down-regulation pathways for p53. Pharmacol Res 37: 83-85, 1998.

4. Osman I, Sherman E, Singh B, Venkatraman E, Zelefsky M, Bosl G, Scher H, Shah J, Shaha A, Kraus D, et al: Alteration of p53 pathway in squamous cell carcinoma of the head and neck: Impact on treatment outcome in patients treated with larynx preservation intent. J Clin Oncol 20: 2980-2987, 2002.

5. Nam TK, Lee JH, Cho SH, Chung IJ, Ahn SJ, Song JY, Yoon MS, Chung WK and Nah BS: Low hMLH1 expression prior to definitive chemoradiotherapy predicts poor prognosis in esophageal squamous cell carcinoma. Cancer Lett 260: 109-117, 2008.

6. Li SW, Wang H, Liu ML, Zhang HB, Xiang YQ, Lv X, Xia WX, Zeng MS, Mai HQ, Hong MH, et al: Positive effect of high RKIP expression on reduced distant metastasis by chemotherapy when combined with radiotherapy in locoregionally advanced nasopharyngeal carcinoma: A prospective study. Med Oncol 30: 322, 2013.

7. Cheng SH, Jian JJ, Tsai SY, Chan KY, Yen LK, Chu NM, Tan TD, Tsou MH and Huang AT: Prognostic features and treatment outcome in locoregionally advanced nasopharyngeal carcinoma following concurrent chemotherapy and radiotherapy. Int J Radiat Oncol Biol Phys 41: 755-762, 1998.

8. Chen C, Chen S, Le QT, Chen J, Chen Z, Li D, Zhou M and Li D: Prognostic model for distant metastasis in locally advanced nasopharyngeal carcinoma after concurrent chemoradiotherapy. Head Neck 37: 209-214, 2015.

9. Wang TL, Diaz LA Jr, Romans K, Bardelli A, Saha S, Galizia G, Choti M, Donehower R, Parmigiani G, Shih IeM, et al: Digital karyotyping identifies thymidylate synthase amplification as a mechanism of resistance to 5-fluorouracil in metastatic colorectal cancer patients. Proc Natl Acad Sci USA 101: 3089-3094, 2004.

10. van den Broek GB, Wreesmann VB, van den Brekel MW, Rasch CR, Balm AJ and Rao PH: Genetic abnormalities associated with chemoradiation resistance of head and neck squamous cell carcinoma. Clin Cancer Res 13: 4386-4391, 2007.

11. Przybytkowski E, Aguilar-Mahecha A, Nabavi S, Tonellato PJ and Basik $\mathrm{M}$ : Ultradense array $\mathrm{CGH}$ and discovery of micro-copy number alterations and gene fusions in the cancer genome. Methods Mol Biol 973: 15-38, 2013.

12. Joo YH, Park SW, Jung SH, Lee YS, Nam IC, Cho KJ, Park JO, Chung YJ and Kim MS: Recurrent loss of the FHIT gene and its impact on lymphatic metastasis in early oral squamous cell carcinoma. Acta Otolaryngol 133: 992-999, 2013.

13. Kim JH, Hu HJ, Yim SH, Bae JS, Kim SY and Chung YJ: CNVRuler: A copy number variation-based case-control association analysis tool. Bioinformatics 28: 1790-1792, 2012.

14. Jung SH, Yim SH, Hu HJ, Lee KH, Lee JH, Sheen DH, Lim MK, Kim SY, Park SW, Kim SH, et al: Genome-wide copy number variation analysis identifies deletion variants associated with ankylosing spondylitis. Arthritis Rheumatol 66: 2103-2112, 2014.

15. Livak KJ and Schmittgen TD: Analysis of relative gene expression data using real-time quantitative PCR and the 2(-Delta Delta C(T)) method. Methods 25: 402-408, 2001.

16. Oddone N, Morgan GJ, Palme CE, Perera L, Shannon J, Wong E, Gebski V and Veness MJ: Metastatic cutaneous squamous cell carcinoma of the head and neck: The Immunosuppression, Treatment, Extranodal spread, and Margin status (ITEM) prognostic score to predict outcome and the need to improve survival. Cancer 115: 1883-1891, 2009.

17. Bernaldo de Quirós S, Merlo A, Secades P, Zambrano I, de Santa María IS, Ugidos N, Jantus-Lewintre E, Sirera R, Suarez C and Chiara MD: Identification of TRPC6 as a possible candidate target gene within an amplicon at 11q21-q22.2 for migratory capacity in head and neck squamous cell carcinomas. BMC Cancer 13: 116, 2013.

18. Shi ZZ, Shang L, Jiang YY, Hao JJ, Zhang Y, Zhang TT, Lin DC, Liu SG, Wang BS, Gong T, et al: Consistent and differential genetic aberrations between esophageal dysplasia and squamous cell carcinoma detected by array comparative genomic hybridization. Clin Cancer Res 19: 5867-5878, 2013.

19. Gao Z, Meng X, Mu D, Sun X and Yu J: Prognostic significance of epidermal growth factor receptor in locally advanced esophageal squamous cell carcinoma for patients receiving chemoradiotherapy. Oncol Lett 7: 1118-1122, 2014.

20. Szabó B, Nelhubel GA, Kárpáti A, Kenessey I, Jóri B, Székely C, Peták I, Lotz G, Hegedus Z, Hegedus B, et al: Clinical significance of genetic alterations and expression of epidermal growth factor receptor (EGFR) in head and neck squamous cell carcinomas. Oral Oncol 47: 487-496, 2011. 
21. Chung CH, Ely K, McGavran L, Varella-Garcia M, Parker J, Parker N, Jarrett C, Carter J, Murphy BA, Netterville J, et al: Increased epidermal growth factor receptor gene copy number is associated with poor prognosis in head and neck squamous cell carcinomas. J Clin Oncol 24: 4170-4176, 2006.

22. Wilson PM, Danenberg PV, Johnston PG, Lenz HJ and Ladner RD: Standing the test of time: Targeting thymidylate biosynthesis in cancer therapy. Nat Rev Clin Oncol 11: 282-298, 2014.

23. Johnston PG, Fisher ER, Rockette HE, Fisher B, Wolmark N, Drake JC, Chabner BA and Allegra CJ: The role of thymidylate synthase expression in prognosis and outcome of adjuvant chemotherapy in patients with rectal cancer. J Clin Oncol 12: 2640-2647, 1994.

24. Murata S, Adachi M, Kioi M, Torigoe S, Ijichi K, Hasegawa Y, Ogawa T, Bhayani MK, Lai SY, Mitsudo K, et al: Etodolac improves 5-FU sensitivity of head and neck cancer cells through inhibition of thymidylate synthase. Anticancer Res 31: 2893-2898, 2011.

25. Yeh KH, Shun CT, Chen CL, Lin JT, Lee WJ, Lee PH, Chen YC and Cheng AL: High expression of thymidylate synthase is associated with the drug resistance of gastric carcinoma to high dose 5-fluorouracil-based systemic chemotherapy. Cancer 82: $1626-1631,1998$

26. Yasumatsu R, Nakashima T, Uryu H, Ayada T, Wakasaki T, Kogo R, Masuda M, Fukushima M and Komune S: Correlations between thymidylate synthase expression and chemosensitivity to 5-fluorouracil, cell proliferation and clinical outcome in head and neck squamous cell carcinoma. Chemotherapy 55: 36-41, 2009.
27. Sunwoo JB, Sun PC, Gupta VK, Schmidt AP, El-Mofty S and Scholnick SB: Localization of a putative tumor suppressor gene in the sub-telomeric region of chromosome $8 \mathrm{p}$. Oncogene 18: 2651-2655, 1999.

28. Ishwad CS, Shuster M, Bockmühl U, Thakker N, Shah P, Toomes C, Dixon M, Ferrell RE and Gollin SM: Frequent allelic loss and homozygous deletion in chromosome band 8p23 in oral cancer. Int J Cancer 80: 25-31, 1999.

29. Toomes C, Jackson A, Maguire K, Wood J, Gollin S, Ishwad C, Paterson I, Prime S, Parkinson K, Bell S, et al: The presence of multiple regions of homozygous deletion at the CSMD1 locus in oral squamous cell carcinoma question the role of CSMD1 in head and neck carcinogenesis. Genes Chromosomes Cancer 37: 132-140, 2003.

30. Ma C, Quesnelle KM, Sparano A, Rao S, Park MS, Cohen MA, Wang Y,Samanta M,Kumar MS, Aziz MU, et al: Characterization CSMD1 in a large set of primary lung, head and neck, breast and skin cancer tissues. Cancer Biol Ther 8: 907-916, 2009.

31. Zhang R and Song C: Loss of CSMD1 or 2 may contribute to the poor prognosis of colorectal cancer patients. Tumour Biol 35: 4419-4423, 2014.

32. Kamal M, Shaaban AM, Zhang L, Walker C, Gray S, Thakker N, Toomes C, Speirs V and Bell SM: Loss of CSMD1 expression is associated with high tumour grade and poor survival in invasive ductal breast carcinoma. Breast Cancer Res Treat 121: 555-563, 2010. 